\title{
Qualidade de vida no Transtorno Obsessivo-Compulsivo: um estudo com usuários da Atenção Básica
}

\author{
Quality of life with Obsessive-Compulsive Disorder: \\ a study among Primary Care users
}

\author{
Carolina Coelho Scholl ${ }^{1}$ \\ Viviane Porto Tabeleão ${ }^{1}$ \\ Rafaelle Stark Stigger ${ }^{2}$ \\ Jéssica Puchalski Trettim ${ }^{1}$ \\ Mariana Bonati de Mattos ${ }^{1}$ \\ Andressa Jacondino Pires ${ }^{1}$ \\ Mariane Acosta Lopez Molina ${ }^{1}$ \\ Ricardo Azevedo da Silva ${ }^{1}$ \\ Elaine Tomasi ${ }^{3}$ \\ Luciana de Avila Quevedo ${ }^{1}$
}

${ }^{1}$ Programa de Pós-

Graduação em Saúde

e Comportamento da

Universidade Católica

de Pelotas (UCPel). R.

Gonçalves Chaves 373/

prédio C/411, Centro.

96015-560 Pelotas

RS Brasil.

lu.quevedo@bol.com.br

${ }^{2}$ Mestrado Profissional em

Saúde da Mulher, Criança e

Adolescente, UCPel. Pelotas

RS Brasil.

${ }^{3}$ Departamento de Medicina Social, UFPel. Pelotas RS

Brasil.
Abstract Quality of life (QOL) can be affected by the presence of mental disorders, like $\mathrm{Ob}$ sessive-Compulsive Disorder (OCD). Thus, the evaluation and monitoring of QOL in patients with mental disorders enables the identification of priorities, making it possible to implement actions to improve QOL among health system users. The scope of this article is to measure QOL in OCD patients in primary health care. It involves a cross-sectional study with a convenience sample including all users of three Basic Health Units of Pelotas in the State of Rio Grande do Sul, Brazil. The quality of life was measured with the WHOQOL-Bref and the OCD was assessed using the Mini-International Neuropsychiatric Interview (M.I.N.I.) This study included 1081 individuals. The prevalence of OCD was 3.9\%. OCD patients had a lower average in all domains of $Q O L$ when compared to individuals without OCD ( $p<$ 0.001). The findings of this study emphasize the importance of using QOL as a monitoring tool of the disorder in basic health care.

Key words Obsessive-compulsive disorder, Quality of life, Primary health care
Resumo A qualidade de vida (QV) pode ser afetada pela presença de transtornos mentais, como o Transtorno Obsessivo-Compulsivo (TOC). Assim, a avaliação e o acompanhamento dos indices de QV em pacientes com transtornos mentais permite a identificação de suas prioridades, sendo possivvel a implementação de ações para a melhora desses índices nos usuários do sistema de saúde. $O$ objetivo deste artigo é avaliar a QV em portadores de TOC usuários da atenção primária à saúde. Estudo transversal com amostragem por conveniência, incluindo todos os usuários de três Unidades Básicas de Saúde de Pelotas, RS. Para avaliar a QV foi utilizada a WHOQOL-Bref, e o TOC foi avaliado através da M.I.N.I. Foram avaliados 1081 indivíduos. A prevalência de TOC foi de 3,9\%. Portadores de TOC apresentaram médias inferiores em todos os dominios da $Q V$ quando comparados aos indivíduos sem TOC $(p<0,001)$. Os achados deste estudo enfatizam a importância de utilizar a $Q V$ como instrumento de monitoramento da melhora do transtorno no âmbito da atenção básica à saúde.

Palavras-chave Transtorno obsessivo-compulsivo, Qualidade de vida, Atenção Primária à Saúde 


\section{Introdução}

De acordo com a Organização Mundial da Saúde (OMS), a qualidade de vida é definida como "a percepção do indivíduo de sua inserção na vida no contexto da cultura e sistemas de valores nos quais ele vive e em relação aos seus objetivos, expectativas, padrões e preocupações" ${ }^{1}$, podendo ser afetada por diversos fatores, entre eles, a presença de transtornos mentais ${ }^{2,3}$.

De todos os transtornos mentais, o Transtorno Obsessivo-Compulsivo (TOC) foi considerado pela OMS como a $10^{\text {a }}$ causa de incapacidade no mundo ${ }^{4-6}$. O TOC é um transtorno caracterizado por ideias obsessivas e/ou por comportamentos compulsivos recorrentes e tem sido reconhecido por seu curso crônico e incapacitante ${ }^{4}$. Tais ideias e comportamentos perturbam muito e consomem tempo da vida do sujeito, interferindo ocupacional e socialmente.

Diversos autores ${ }^{5,7-13}$ citam como consequências comuns do TOC a diminuição da autoestima e do bem-estar subjetivo e a interferência negativa na vida estudantil, profissional, familiar, afetiva e social. Em casos mais graves pode haver uma completa dificuldade ou incapacidade para atividades rotineiras e alguns portadores podem ficar totalmente presos em casa, em função de comportamentos de esquiva dos estímulos temidos ${ }^{5}$.

Estudos populacionais mostram que a qualidade de vida no TOC é menor quando comparada à população geral ${ }^{7,14,15}$. Os sintomas do transtorno influenciam negativamente nas atividades diárias e sociais, causando pior qualidade de vida relacionada à saúde mental e acarretando altas prevalências de ideação e tentativas de suicídio ao longo da vida ${ }^{5}$. Entretanto, estudo com pacientes ambulatoriais ${ }^{16}$ não encontrou diferenças na qualidade de vida relacionadas à casamento, abuso de álcool e tentativas de suicídio, mas índices indiretos, como pior desempenho de papéis sociais e funcionamento social, mais desemprego e maior proporção de solteiros.

A avaliação e o acompanhamento dos índices de qualidade de vida em pacientes com transtornos mentais permite a identificação de suas prioridades, sendo possível a implementação de ações efetivas para melhorar a dos usuários do sistema de saúde $e^{17,18}$. A atenção primária é a porta de entrada da atenção à saúde no Brasil, sendo definida como estratégia de organização do sistema para realizar ações de promoção à saúde, prevenção de agravos, diagnóstico, tratamento e reabilitação individual e coletiva ${ }^{19}$. Entretanto, estudos com sujeitos atendidos na atenção pri- mária são escassos e aqueles relacionados especificamente ao TOC são inexistentes.

Assim, o objetivo deste estudo foi avaliar a qualidade de vida em portadores de TOC usuários da atenção primária à saúde.

\section{Métodos}

Trata-se de um estudo transversal aninhado a um de intervenção conduzido nas quatro unidades de básicas de saúde (UBS) vinculadas à Universidade Católica de Pelotas no município de Pelotas (zona sul do estado do Rio Grande do Sul). Na primeira fase, o trabalho consistiu na avaliação dos modelos de diagnóstico e tratamento de transtornos mentais realizados por médicos e estudantes de medicina nas UBS referidas.

$\mathrm{Na}$ segunda fase, foi realizado treinamento de modelo de entrevista breve para avaliar os transtornos psiquiátricos nos pacientes atendidos e formas de intervenção e encaminhamento. Em paralelo, foram obtidas as prevalências de transtornos mentais e comorbidades nos usuários dos serviços. No período do estudo, os médicos e os estudantes, além de preencherem a ficha de atendimento, responderam a um instrumento sobre informações relacionadas às doenças físicas e presença de transtorno mental e a forma de tratamento escolhida. A equipe de pesquisa colheu estes dados enquanto psicólogos e psiquiatras treinados no uso do instrumento visitaram as residências para aplicação dos mesmos. Nesta mesma visita, o paciente respondeu a questões referentes a dados sociodemográficos e uso de recursos de saúde. Seis meses após o treinamento, a equipe de pesquisa realizou novamente os procedimentos da primeira etapa, avaliando igualmente o diagnóstico e as formas de tratamento.

A amostra foi selecionada por conveniência, incluindo todos os usuários das três UBS que buscaram algum tipo de atendimento no período de $1^{\circ}$ de março a 30 de julho de 2009. Foram considerados elegíveis os indivíduos maiores de 14 anos moradores da área de abrangência de cada UBS. Os dados de identificação foram obtidos do prontuário do usuário no dia do atendimento. Posteriormente, com base no endereço registrado no prontuário, entrevistadores capacitados realizaram visitas domiciliares para a aplicação dos instrumentos.

Para o presente estudo foi utilizada o World Health Organization Quality of Life Assessment (WHOQOL - Bref $)^{1,20}$, instrumento composto por 26 questões relacionadas à qualidade de vida 
nas duas últimas semanas e agrupadas em quatro domínios: saúde física, psicológica, relações sociais e meio ambiente. A avaliação foi verificada através de médias dos escores de cada domínio, variando de 0 a 100. Quanto mais altas as médias, melhor percepção de qualidade de vida tem o indivíduo.

O Transtorno Obsessivo-Compulsivo foi avaliado através da Mini International Neuropsychiatric Interview (M.I.N.I. $)^{21,22}$, uma entrevista diagnóstica semiestruturada e padronizada de acordo com os critérios da quarta versão do Manual Diagnóstico e Estatístico de Transtornos Mentais (DSM-IV) e a 10a revisão da Classificação Estatística Internacional de Doenças e Problemas Relacionados à Saúde (CID-10), gerando diagnósticos clínicos dos principais transtornos mentais. É dividida em módulos independentes, com cada um correspondendo a um transtorno. As questões sobre o TOC referem-se aos sintomas presentes no último mês.

A detecção do uso diário de álcool e tabaco foi realizada através do Alcohol, Smoking and Substance Involvement Screening Test (ASSIST) ${ }^{23,24}$, questionário estruturado composto por oito questões sobre o uso de nove classes de substâncias psicoativas nos últimos três meses: tabaco, álcool, maconha, cocaína, estimulantes, sedativos, inalantes, alucinógenos e opiáceos. Por sua maior ocorrência, optou-se incluir apenas o uso de tabaco e álcool nas análises.

Além dos instrumentos citados, também foi utilizado um questionário com informações sobre características sociodemográficas e comportamentais, como: sexo, idade (em tercis), escolaridade e classe socioeconômica. Esta última foi classificada de acordo com a Associação Brasileira de Empresas de Pesquisa $(\mathrm{ABEP})^{25}$, a qual se baseia no acúmulo de bens materiais e na escolaridade do chefe da família, classificando-o em cinco níveis (A, B, C, D e E), sendo o nível A, a classe mais alta, e o nível $\mathrm{E}$, a mais baixa.

Os dados foram analisados utilizando o programa SPSS 13.0. Para comparar as médias de qualidade de vida e as variáveis independentes foram utilizados o teste t de student, a ANOVA e Bonferroni. Para as análises dos principais fatores associados à qualidade de vida, foi utilizado um modelo hierárquico no qual foram incluídas as variáveis que apresentaram valor de $\mathrm{p}<0,20$ nas análises univariadas antes mencionadas ${ }^{26}$. No primeiro nível foram incluídas as variáveis sociodemográficas e econômicas; o segundo foi composto pelas comportamentais; e o terceiro, pelo Transtorno Obsessivo-Compulsivo. Para a análise multivariável deste modelo, foi realizada regressão linear múltipla, calculando o e o intervalo de confiança de 95\% (IC95\%) de cada uma das variáveis de exposição analisadas. Adicionalmente, foi calculado o R2 de cada nível para comparar quanto cada um deles contribuiu para explicar a variabilidade do desfecho.

Todos os participantes assinaram um termo de consentimento livre e esclarecido, sendo que para os menores de 18 anos foi solicitada, no termo de consentimento, a assinatura do responsável. O projeto foi aprovado pelo Comitê de Ética em Pesquisa da UCPel.

\section{Resultados}

Foram identificados 1518 pacientes, entretanto, entre a data de identificação e a entrevista nas residências, ocorreram 437 (28,8\%) perdas, sendo incluídos na amostra 1081 indivíduos. A maior parte dos sujeitos era do sexo feminino $(71,3 \%)$, com idades entre 38 e 57 anos $(34,1 \%)$, pertencente à classe econômica $\mathrm{C}(60,7 \%)$ e que vivia com companheiro(a) $(54,0 \%)$. O uso diário de tabaco foi referido por $26,4 \%$ dos indivíduos, e o de álcool, por 17,3\%. A prevalência de TOC foi de 3,9\% $(\mathrm{n}=42)$ (Tabela 1$)$.

$\mathrm{Na}$ análise bruta, os homens demonstraram melhor qualidade de vida nos domínios físico ( $p$ $=0,005)$, psicológico $(\mathrm{p}=0,001)$ e relações sociais $(p=0,013)$ comparados às mulheres. A idade foi associada com todos os domínios da qualidade de vida, sendo que pessoas com idades de entre 38 e 57 anos apresentaram médias inferiores nos psicológico ( $\mathrm{p}<0,001)$, relações sociais ( $\mathrm{p}<$ $0,001)$ e ambiente $(\mathrm{p}<0,001)$. Já indivíduos com 58 anos ou mais evidenciaram médias inferiores no domínio físico $(\mathrm{p}<0,001)$. Com relação à classe econômica, os indivíduos das classes D e E mostraram menores médias em todos os domínios do WHOQOL-Bref $(\mathrm{p}<0,001)$. Também foram evidenciadas médias inferiores nos domínios psicológico $(\mathrm{p}=0,011)$, relações sociais $(\mathrm{p}<$ $0,001)$ e ambiente $(\mathrm{p}<0,001)$ entre os indivíduos que não viviam com companheiro(a). Fumar diariamente foi relacionado à menor qualidade de vida em todos os domínios ( $\mathrm{p} \leq 0,001)$, exceto domínio físico. O uso diário de álcool esteve associado a maiores médias nos domínios físico $(\mathrm{p}<0,001)$ e psicológico $(\mathrm{p}=0,002)$. Portadores de TOC apresentaram médias inferiores em todos os domínios da qualidade de vida quando comparados aos indivíduos sem TOC $(\mathrm{p}<0,001)$ (Tabela 2). 
Tabela 1. Características da amostra de pacientes de três Unidades Básicas de Saúde de Pelotas.

\begin{tabular}{lcc}
\hline \multicolumn{1}{c}{ Variável } & N & $\%$ \\
\hline Sexo & & \\
Masculino & 310 & 28,7 \\
Feminino & 771 & 71,3 \\
Idade & & \\
14-37 & 355 & 32,8 \\
$38-57$ & 369 & 34,1 \\
58 ou mais & 357 & 33,0 \\
Classificação econômica* & & \\
A e B & 111 & 10,4 \\
C & 646 & 60,7 \\
D e E & 308 & 28,9 \\
Viver com companheiro(a) & & \\
Não & 503 & 46,0 \\
Sim & 577 & 54,0 \\
Uso diário de tabaco* & & \\
Não & 796 & 73,6 \\
Sim & 285 & 26,4 \\
Uso diário de álcool & & \\
Não & & 82,7 \\
Sim & 894 & 17,3 \\
TOC & 187 & \\
Não & & \\
Sim & 1039 & 96,1 \\
Total & 42 & 3,9 \\
\hline
\end{tabular}

*Variáveis com missing.

$\mathrm{Na}$ análise ajustada, as variáveis sexo e idade permaneceram associadas aos domínios aspecto físico ( $\mathrm{p}=0,004 ; \mathrm{p}<0,001$, respectivamente), aspecto psicológico ( $\mathrm{p}=0,001 ; 0,036$ respectivamente) e sexo às relações sociais $(\mathrm{p}=0,035)$. A variável classificação econômica se manteve associada a todos os domínios do WHOQOL-Bref ( $\mathrm{p}$ $<0,001$ ); viver com companheiro(a) ao domínio relações sociais $(\mathrm{p}=0,001)$; tabaco aos domínios aspecto psicológico $(\mathrm{p}<0,010)$, relações sociais $(\mathrm{p}=0,016)$ e ambiente $(\mathrm{p}=0,001)$. Uso diário de álcool se manteve associado ao aspecto físico ( $\mathrm{p}$ $=0,003)$, e TOC permaneceu associado a todos os domínios do WHOQOL-Bref $(\mathrm{p}<0,001)$. Homens tiveram 3,9 ( $\beta=3$,9 IC 95\% 1,$3 ; 6,5)$ pontos a mais na média do domínio aspecto físico quando comparados às mulheres; 3,7 ( $\beta=3,7$ IC $95 \% 1,4 ; 5,9)$ pontos a mais no domínio aspecto psicológico e 2,5 ( $\beta=2,5$ IC 95\% 0,2; 4,8) pontos a mais no domínio relações sociais. A média diminuiu 7,5 ( $\beta=-7,5$ IC 95\% -8,9; -6,0) pontos no domínio aspecto físico e 1,3 ( $\beta=-1,3$ IC $95 \%$ $-2,6 ;-0,9)$ pontos no domínio aspecto psicológi- co à medida que a idade aumentava. Com relação à classificação socioeconômica, enquanto esta diminuía, de A para $\mathrm{E}$, as médias reduziram 5,5 ( $\beta$ $=-5,5$ IC $95 \%-7,5 ;-3,8)$ pontos no domínio aspecto físico, 5,3 ( $\beta=-5,3$ IC 95\% -7, $1 ;-3,5)$ pontos no domínio aspecto psicológico, 4,6 ( $\beta=-4,6$ IC $95 \%-6,4 ;-2,8)$ no domínio relações sociais e 5,6 ( $\beta=-5,6$ IC 95\% -7,0;-4,2) pontos no domínio ambiente. Indivíduos que viviam com companheiro(a) tiveram 3,5 ( $\beta=3,5$ IC 95\% 1,4; 5,7) pontos a mais no domínio relações sociais do que aqueles que viviam sem companheiro(a). Indivíduos que usavam tabaco diariamente tiveram 4,5 $(\beta=-4,5$ IC $95 \%-6,8 ;-2,1)$ pontos a menos na média do domínio psicológico, 2,9 ( $\beta=-2,9$ IC $95 \%-5,3 ;-0,5)$ pontos a menos na média do domínio relações sociais e 3,2 ( $\beta=-3,2$ IC 95\% -5,1; $-1,3)$ pontos a menos no domínio ambiente. $\mathrm{O}$ uso de álcool somente permaneceu significativo no aspecto físico, com um aumento de 4,9 ( $\beta=$ $4,9$ IC 95\% 1,$7 ; 8,2)$ pontos na média. Portadores de TOC tiveram redução de 23,8 $(\beta=-23,8$ IC $95 \%-29,7 ;-17,9)$ pontos na média do domínio físico quando comparados àqueles sem TOC, redução de 27,3 ( $\beta=-27,3$ IC 95\% -32,4; -22,2) pontos na média do domínio psicológico, 12,6 ( $\beta$ $=-12,6$ IC $95 \%-17,9 ;-7,3)$ pontos no domínio relações sociais e 14,9 ( $\beta=-14,9$ IC $95 \%-19,1$; -10,6) pontos no domínio ambiente (Tabela 3 ).

A Tabela 3 também mostra que o nível 3 explica $19 \%, 16 \%, 7 \%$ e $11 \%$ da variabilidade dos aspectos físico, psicológico, relações sociais e ambiente, respectivamente. Para todos os desfechos, o nível 3 explicou uma proporção maior da variabilidade quando comparado com os outros, especialmente no aspecto psicológico, que passou de $7 \%$ para $16 \%$.

\section{Discussão}

Este estudo verificou que indivíduos com TOC apresentaram médias inferiores em todos os domínios da qualidade de vida, corroborando com os dados da OMS que mencionam este transtorno como um dos mais incapacitantes. Diversos estudos também mostram menores médias de qualidade de vida no TOC quando comparados à população em geral ${ }^{2,7,14,15,27,28}$. Os impactos negativos deste transtorno trazem diversos prejuízos na vida dos sujeitos, afetando diversas áreas ${ }^{14}$, como, por exemplo, a capacidade de trabalhar e desempenhar atividades diárias, de se relacionar com outras pessoas e de desfrutar das atividades de lazer ${ }^{7}$. A partir disso, os indivíduos relatam 
Tabela 2. Distribuição da amostra e médias do WHOQOL - Bref em pacientes atendidos em três Unidades Básicas de Saúde de Pelotas (RS).

\begin{tabular}{|c|c|c|c|c|c|c|c|c|}
\hline Variável & $\begin{array}{l}\text { AF Média } \\
( \pm d p)\end{array}$ & p-valor & $\begin{array}{l}\text { AP Média } \\
( \pm d p)\end{array}$ & p-valor & $\begin{array}{l}\text { RS Média } \\
( \pm \mathrm{dp})\end{array}$ & p-valor & $\begin{array}{c}\text { Ambiente } \\
\text { Média }( \pm \mathrm{dp})\end{array}$ & p-valor \\
\hline Sexo & & 0,005 & & 0,001 & & 0,013 & & 0,099 \\
\hline Masculino & $67,3( \pm 21,6)$ & & $69,5( \pm 17,4)$ & & $72,9( \pm 17,2)$ & & $59,8( \pm 14,8)$ & \\
\hline Feminino & $63,4( \pm 20,5)$ & & $65,5( \pm 17,3)$ & & $70,0( \pm 17,9)$ & & $58,2( \pm 13,9)$ & \\
\hline Idade & & $<0,001$ & & $<0,001$ & & $<0,001$ & & $<0,001$ \\
\hline $14-37$ & $74,4( \pm 16,8)$ & & $70,1( \pm 16,7)$ & & $73,9( \pm 19,2)$ & & $61,6( \pm 14,2)$ & \\
\hline $38-57$ & $61,1( \pm 21,6)$ & & $63,8( \pm 18,5)$ & & $68,2( \pm 18,2)$ & & $55,3( \pm 14,4)$ & \\
\hline 58 ou mais & $58,0( \pm 20,0)$ & & $66,1( \pm 16,2)$ & & $70,4( \pm 15,1)$ & & $59,6( \pm 13,3)$ & \\
\hline Classificação & & $<0,001$ & & $<0,001$ & & $<0,001$ & & $<0,001$ \\
\hline econômica & $73,9( \pm 18,8)$ & & $74,3( \pm 14,1)$ & & $79,3( \pm 15,8)$ & & $66,8( \pm 12,8)$ & \\
\hline A e B & $65,9( \pm 19,9)$ & & $67,6( \pm 17,2)$ & & $71,2( \pm 17,2)$ & & $59,3( \pm 13,9)$ & \\
\hline $\mathrm{C}$ & $58,4( \pm 21,8)$ & & $62,1( \pm 17,7)$ & & $67,1( \pm 18,5)$ & & $54,4( \pm 13,8)$ & \\
\hline D e E & & & & & & & & \\
\hline Viver com & & 0,058 & & 0,011 & & $<0,001$ & & 0,001 \\
\hline companheiro(a) & $63,1( \pm 21,5)$ & & $65,2( \pm 18,1)$ & & $68,2( \pm 18,8)$ & & $57,1( \pm 14,5)$ & \\
\hline Não & $65,6( \pm 20,2)$ & & $67,9( \pm 16,7)$ & & $73,0( \pm 16,5)$ & & $59,9( \pm 13,9)$ & \\
\hline Sim & & & & & & & & \\
\hline Uso diário de & & 0,237 & & 0,001 & & 0,001 & & $<0,001$ \\
\hline tabaco & $64,9( \pm 20,6)$ & & $67,8( \pm 16,6)$ & & $71,9( \pm 17,7)$ & & $59,7( \pm 14,0)$ & \\
\hline Não & $63,2( \pm 21,7)$ & & $63,5( \pm 19,1)$ & & $67,8( \pm 17,7)$ & & $55,7( \pm 14,5)$ & \\
\hline $\operatorname{Sim}$ & & & & & & & & \\
\hline Uso diário de & & $<0,001$ & & 0,002 & & 0,226 & & 0,069 \\
\hline álcool & $63,0( \pm 21,0)$ & & $65,9( \pm 17,4)$ & & $70,5( \pm 17,6)$ & & $58,2( \pm 14,2)$ & \\
\hline Não & $71,5( \pm 18,7)$ & & $70,2( \pm 16,7)$ & & $72,2( \pm 18,2)$ & & $60,4( \pm 14,1)$ & \\
\hline$\underset{\text { TOC }}{\text { Sim }}$ & & $<0,001$ & & $<0,001$ & & $<0,001$ & & $<0,001$ \\
\hline Não & $65,4( \pm 20,5)$ & & $67,7( \pm 16,5)$ & & $71,3( \pm 17,4)$ & & $59,3( \pm 13,9)$ & \\
\hline Sim & $41,8( \pm 17,4)$ & & $39,4( \pm 16,7)$ & & $58,1( \pm 21,5)$ & & $43,1( \pm 13,0)$ & \\
\hline Total & $64,5( \pm 20,9)$ & & $66,6( \pm 17,4)$ & & $70,8( \pm 17,7)$ & & $58,6( \pm 14,2)$ & \\
\hline
\end{tabular}

${ }^{*}$ Diferenças significativas pelo teste Bonferroni. AF - Aspecto Físico; AP - Aspecto Psicológico; RS - Relações Sociais

tais prejuízos, demonstrando a piora em sua qualidade de vida.

Comparando as médias de todos os domínios da qualidade de vida, o aspecto psicológico foi o mais afetado entre os portadores de TOC. Isso pode ser ocasionado devido aos sentimentos negativos e à sensação de "perda de controle" que as obsessões e compulsões bizarras e incompreensíveis causam ${ }^{28}$. Cabe ressaltar que o TOC não só se mostrou associado aos domínios da qualidade de vida, como também explicou a maior variabilidade, principalmente no aspecto psicológico, reafirmando a importância da sua influência na qualidade de vida.

Seguinte a esse, o domínio aspecto físico apresentou médias inferiores de qualidade de vida. Moritz et al. ${ }^{11}$ relatam em seu estudo que as compulsões de limpeza podem ocasionar problemas dermatológicos, enquanto que outras podem promover exaustão física. Entretanto, controverso ao nosso resultado, encontra-se na literatura que o domínio físico é, tipicamente, o menos afetado em indivíduos com TOC $^{27,28}$. Isso pode ter ocorrido porque nossa amostra contém uma importante prevalência de idosos e, como citamos anteriormente, pessoas com mais de 57 anos apresentaram médias inferiores no aspecto físico.

Stengler-Wenzke et al. ${ }^{28}$ apontaram que o segundo domínio mais prejudicado é o de relações sociais, enquanto Srivastava et al. ${ }^{29}$ encontraram que este seria o mais afetado, ambos resultados diferem dos nossos. Tal disparidade pode ser explicada pelas diferenças culturais e comportamentais entre as amostras, pois as duas pesquisas foram realizadas em países diferentes do Brasil. E também, isso pode ter ocorrido devido ao fato de amigos e familiares dos portadores de TOC, com frequência, se acomodarem aos sintomas, auxiliando-os a perpetuar pensamentos e comportamentos derivados do transtorno. Dessa maneira, pode não haver interferências negativas em seu relacionamento. 
Tabela 3. Regressão linear entre as características da amostra e médias do WHOQOL-Bref.

\begin{tabular}{|c|c|c|c|c|c|c|}
\hline Variável & $\begin{array}{c}\mathrm{AF} \\
(\mathrm{IC} 95 \%)\end{array}$ & $\begin{array}{c}\mathrm{R} 2 \\
\text { ajustado }\end{array}$ & p-valor & $\begin{array}{c}\text { AP } \\
(\text { IC } 95 \%)\end{array}$ & $\begin{array}{c}\mathrm{R} 2 \\
\text { ajustado }\end{array}$ & p-valor \\
\hline $1^{\circ}$ nível & & 0,13 & & & 0,05 & \\
\hline Sexo & $3,9(1,3 ; 6,5)$ & & 0,004 & $3,7(1,4 ; 5,9)$ & & 0,001 \\
\hline Idade & $-7,5(-8,9 ;-6,0)$ & & $<0,001$ & $-1,3(-2,6 ;-0,9)$ & & 0,036 \\
\hline $\begin{array}{l}\text { Classificação Socioeconômica } \\
\text { (D e E) }\end{array}$ & $-5,5(-7,5 ;-3,8)$ & & $<0,001$ & $-5,3(-7,1 ;-3,5)$ & & $<0,001$ \\
\hline Viver com companheiro(a) & $1,1(-1,2 ; 3,5)$ & & 0,351 & $1,2(-0,8 ; 3,3)$ & & 0,246 \\
\hline 2 nível & & 0,14 & & & 0,06 & \\
\hline Uso diário de tabaco & --- & & --- & $-4,5(-6,8 ;-2,1)$ & & $<0,001$ \\
\hline Uso diário de álcool & $4,9(1,7 ; 8,2)$ & & 0,003 & $3,3(0,5 ; 6,1)$ & & 0,147 \\
\hline 3o nível & & 0,19 & & & 0,16 & \\
\hline TOC & $-23,8(-29,7 ;-17,9)$ & & $<0,001$ & $-27,3(-32,4 ;-22,2)$ & & $<0,001$ \\
\hline Variável & $\begin{array}{c}\text { RS } \beta \\
\text { (IC 95\%) }\end{array}$ & $\begin{array}{c}\mathrm{R} 2 \\
\text { ajustado }\end{array}$ & p-valor & $\begin{array}{l}\text { Ambiente } \\
\text { (IC 95\%) }\end{array}$ & $\begin{array}{c}\mathrm{R} 2 \\
\text { ajustado }\end{array}$ & p-valor \\
\hline $1^{\circ}$ nível & & 0,05 & & & 0,06 & \\
\hline Sexo & $2,5(0,2 ; 4,8)$ & & 0,035 & $1,3(-0,5 ; 3,2)$ & & 0,151 \\
\hline Idade & $-1,2(-2,5 ;-0,7)$ & & 0,064 & $-0,6(-1,1 ; 0,9)$ & & 0,902 \\
\hline $\begin{array}{l}\text { Classificação } \\
\text { Socioeconômica (D e E) }\end{array}$ & $-4,6(-6,4 ;-2,8)$ & & $<0,001$ & $-5,6(-7,0 ;-4,2)$ & & $<0,001$ \\
\hline Viver com companheiro(a) & $3,5(1,4 ; 5,7)$ & & 0,001 & $1,4(-0,3 ; 3,1)$ & & 0,107 \\
\hline $2^{\circ}$ nível & & 0,06 & & & 0,07 & \\
\hline Uso diário de tabaco & $-2,9(-5,3 ;-0,5)$ & & 0,016 & $-3,2(-5,1 ;-1,3)$ & & 0,001 \\
\hline Uso diário de álcool & --- & & --- & --- & & --- \\
\hline $3^{\circ}$ nível & & 0,07 & & & 0,11 & \\
\hline TOC & $-12,6(-17,9 ;-7,3)$ & & $<0,001$ & $-14,9(-19,1 ;-10,6)$ & & $<0,001$ \\
\hline
\end{tabular}

AF - Aspecto Físico; AP - Aspecto Psicológico; RS - Relações Sociais.

Não existem muitos estudos que descrevam a relação entre os domínios do WHOQOL-Bref em indivíduos com TOC e as variáveis sociodemográficas. O estudo de Stengler-Wenzke et al. ${ }^{28}$ corrobora com um de nossos resultados, afirmando que quanto mais velhas as pessoas, pior sua qualidade de vida relacionada a todos os domínios, exceto ambiente. Além disso, algumas pesquisas evidenciam que portadores de TOC geralmente apresentam maior taxa de desemprego, menor nível socioeconômico, menor índice de casamento ou união conjugal estável e vivem mais sozinhos ${ }^{30-32}$, afirmando que essas variáveis estão fortemente associadas à qualidade de vida ${ }^{7,33}$.

Cabe destacar que, por ser um estudo com amostragem por conveniência, não se pode afirmar que há representatividade na população em geral. Também, deve-se pensar como limitação a possibilidade de causalidade reversa, pois não se sabe se o TOC leva a uma pior qualidade de vida ou se esta leva ao TOC. Entretanto, este trabalho serve como subsídio para novos estudos da área.

A partir dos achados do presente estudo, supõe-se que a qualidade de vida é influenciada negativamente pelo transtorno mental, o qual apresenta altas prevalências na atenção primária. Em uma amostra de pacientes da atenção básica de uma cidade na Carolina do Norte, EUA, foram encontrados quase $40 \%$ de sintomas de ansiedade e $30 \%$ de sintomas depressivos ${ }^{33}$. Estudo realizado em uma cidade do Rio Grande do Sul encontrou uma prevalência de 51,1\% de transtornos mentais em indivíduos que buscaram atendimento na atenção primária ${ }^{34}$.

No ano de 2000, a OMS propôs que os atendimentos em saúde mental fossem direcionados para os centros de atendimento primário, com o objetivo de abranger uma parte maior da população ${ }^{34-36}$. Com isso, enfatiza-se a importância de se utilizar a qualidade de vida como instrumento de monitoramento da melhora do transtorno no âmbito da atenção básica à saúde. Os tratamentos existentes possuem efeitos positivos, não só 
na melhora ou diminuição dos sintomas obsessivo-compulsivos, como também na qualidade de vida do paciente, minimizando seu sofrimento e o impacto da doença.

\section{Colaboradores}

CC Scholl e LA Quevedo participaram da análise dos dados, redação do artigo e aprovação da versão a ser publicada.VP Tabeleão e MAL Molina participaram da coordenação do estudo, revisão crítica do artigo e aprovação da versão a ser publicada. RS Stigger, JP Trettim, MB Mattos e AJ Pires participaram da revisão crítica do artigo e da aprovação da versão a ser publicada. RA Silva e E Tomasi participaram da elaboração do projeto, coordenação do estudo, revisão crítica do artigo e aprovação da versão a ser publicada.

\section{Referências}

1. World Health Organization (WHO). The World Health Organization Quality of Life Assessment (WHOQOL): position paper from the World Health Organization. Soc Sci Med 1995; 41(10):403-409.

2. Rapaport MH, Clary C, Fayyad R, Endicott J. Quality-of-life impairment in depressive and anxiety disorders. Am J Psychiatry 2005; 162(6):1171-1178.

3. Roberts J, Lenton P, Keetharuth AD, Brazier J. Quality of life impact of mental health conditions in England: results from the adult psychiatric morbidity surveys. Health Qual Life Outcomes 2014; 12:6.

4. Macy AS, Theo JN, Kaufmann SC, Ghazzaoui RB, Pawlowski PA, Fakhry HI, Cassmassi BJ, IsHak WW. Quality of life in obsessive compulsive disorder. CNS Spectr $2013 ; 18(1): 21-33$

5. Torresan RC, Smaira SI, Ramos-Cerqueira ATA, Torres AR. Qualidade de vida no transtorno obsessivo-compulsivo: uma revisão. Rev Psiq Clin 2008; 35(1):13-19.

6. World Health Organization (WHO). Regional Office for the Eastern Mediterranean. The newly defined burden of mental problems. East Mediterr Health J 1999; 5(2):394-401.

7. Eisen JL, Mancebo MA, Pinto A, Coles ME, Pagano ME, Stout R, Rasmussen SA. Impact of obsessive-compulsive disorder on quality of life. Compr Psychiatry 2006; 47(4):270-275.

8. Hollander E, Know JH, Stein DJ, Broatch J, Rowland T, Himelein CA. Obsessive-compulsive and sprectrum disorders: overview and quality of life issues. J Clin Psychiatry 1996; 57(Supl. 8):3-6.

9. Hollander E, Stein DJ, Know JH, Rowland T, Wong CM, Broatch J. Psychossocial function and economic costs of obsessive-compulsive disorder. CNS Spectr 1997; 2:16.

10. Lochner C, Mogotsi M, du Toit PL, Kaminer D, Niehaus DJ, Stein DJ. Quality of life in anxiety disorders: a comparison of obsessive-compulsive disorder, social anxiety disorder, and panic disorder. Psychopathol 2003; 36(5):255-262.

11. Moritz S, Rufer M, Fricke S, Karow A, Morfeld M, Jelinek L, Jacobsen D. Quality of life in obsessive-compulsive disorder before and after treatment. Compr Psychiatry 2005; 46(6):453-459.

12. Stein DJ, Allen A, Bobes J, Eisen JL, Figuera ML, Koran LM. Quality of life in obsessive-compulsive disorder. CNS Spectr 2000; 5(6 [Supl. 4]):37-39.

13. Steketee G, Grayson JB, Foa EB. A comparison of characteristics of obsessive-compulsive disorder and other anxiety disorders. J Anxiety Disord 1987; 1(4):325-335. 
14. Bobes J, Gonzáles MP, Bascaran MT, Arango C, Saiz PA, Bousono M. Quality of life and disability in patients with obsessive-compulsive disorder. Eur Psychiatry 2001; 16(4):239-245.

15. Rodriguez-Salgado B, Dolengevich-Segal H, Arrojo-Romero M, Castelli-Candia P, Navio-Acosta M, Perez-Rodriguez MM, Saiz-Ruiz J, Baca-Garcia E. Perceived quality of life in obsessive-compulsive disorder: related factors. BMC Psychiatry 2006; 6:20.

16. Koran LM, Thienemann ML, Davenport R. Quality of life for patients with obsessive-compulsive disorder. Am J Psychiatry 1996; 153(6):783-788.

17. Kluthcovsky A, Kluthcovsky F. O WHOQOL-bref, um instrumento para avaliar qualidade de vida: uma revisão sistemática. Rev Psiq Rio Grande do Sul 2009; 31(3):1-12.

18. Skevington SM, Lotfy M, O'Connell KA; WHOQOL Group. The World Health Organization's WHOQOL-BREF quality of life assessment: Psychometric properties and results of the international field Trial a Report from the WHOQOL Group. Qual Life Res 2004; 13(2):299-310

19. Facchini L. Avaliação de efetividade da Atenção Básica à Saúde em municípios das regiões Sul e Nordeste do Brasil: contribuições metodológicas. Cad Saude Publica 2008; 24(Supl. 1):159-172.

20. Fleck MPA, Louzada S, Xavier M, Chachamovich E, Vieira G, Santos L, Pinzon V. Aplicação da versão em português do instrumento abreviado de avaliação da qualidade de vida "WHOQOL-bref". Rev Saude Publica 2000; 34(2):178-183.

21. Amorim P. Mini International Neuropsychiatric Interview (MINI): validação de entrevista breve para diagnóstico de transtornos mentais. Rev Bras Psiquiatr 2000; 22(3):106-115.

22. Sheehan DV, Lecrubier Y, Sheehan KH, Amorim P, Janavs J, Weiller E, Hergueta T, Baker R, Dunbar GC. The Mini-International Neuropsychiatric Interview (M.I.N.I.): the development and validation of a structured diagnostic psychiatric interview for DSM-IV and ICD-10. J Clin Psychiatry 1998; 59(Supl. 20):22-33.

23. Humeniuk R, Ali R, Babor TF, Farrell M, Formigoni ML, Jittiwutikarn J, de Lacerda RB, Ling W, Marsden J, Monteiro M, Nhiwatiwa S, Pal H, Poznyak V, Simon S. Validation of the alcohol, smoking and substance involvement screening test (ASSIST). Addiction 2008; 103(6):1039-1047.

24. Henrique I, Micheli D, Lacerda R, Lacerda L, Formigoni M. Validação da versão brasileira do teste de triagem do envolvimento com álcool, cigarro e outras substâncias (ASSIST). Rev Assoc Med Bras 2004; 50(2):199-206.

25. Associação Brasileira de Empresas de Pesquisa (ABEP). Critério de classificação econômica Brasil. São Paulo: ABEP; 2015.
26. Victora CG, Huttly SR, Fuchs SC, Olinto MT. The role of conceptual frameworks in epidemiological analysis: a hierarchical approach. Int J Epidemiol 1997; 26(1):224-227.

27. Hertenstein E, Thiel N, Herbst N, Freyer T, Nissen C, Külz AK, Voderholzer U. Quality of life changes following inpatient and outpatient treatment in obsessive-compulsive disorder: a study with 12 months follow-up. Ann Gen Psychiatry 2013; 12(1):4.

28. Stengler-Wenzke K, Kroll M, Matschinger H, Angermeyer MC. Subjective quality of life of patients with obsessive-compulsive disorder. Soc Psychiatry Psychiatr Epidemiol 2006; 41(8):662-668.

29. Srivastava S, Bhatia MS, Thawani R, Jhanjee A. Quality of life in patients with obsessive compulsive disorder: A longitudinal study from India. Asian J Psychiatr 2011; 4(3):178-182.

30. Karno M, Golding JM, Sorenson SB, Burnam MA. The epidemiology of obsessive-compulsive disorder in five US communities. Arch Gen Psychiatry 1988; 45(12):1094-1099.

31. Leon AC, Portera L, Weissman MM. The social costs of anxiety disorders. Br J Psychiatry Supl 1995; (27):19-22.

32. Torres AR, Prince MJ, Bebbington PE, Bhugra D, Brugha TS, Farrell M, Jenkins R, Lewis G, Meltzer H, Singleton N. Obsessive-compulsive disorder: prevalence, comorbidity, impact, and help-seeking in the British National Psychiatric Morbidity Survey of 2000. Am J Psychiatry 2006; 163(11):1978-1985.

33. Brenes GA. Anxiety, depression, and quality of life in primary care patients. J Clin Psychiatry 2007; 9(6):437443.

34. Gonçalves DM, Kapczinski F. Prevalência de transtornos mentais em indivíduos de uma unidade de referência para Programa Saúde da Família em Santa Cruz do Sul, Rio Grande do Sul, Brasil. Cad Saude Publica 2008; 24(9):2043-2053.

35. World Health Organization (WHO). Cross-national comparisons of the prevalence and correlates of mental disorders. Bulletin of the World Health Organization 2000; 78(4):413-426.

36. Kohn R, Saxena S, Levav I, Saraceno B. The treatment gap in mental health care. Bulletin of the World Health Organization 2004; 82(11):858-871.

Artigo apresentado em 05/03/2015

Aprovado em 23/10/2015

Versão final apresentada em 25/10/2015 